MDPI

sciforum
MOL2NET, International Conference Series on Multidisciplinary Sciences

MOL2NET 2021, International Conference on Multidisciplinary Sciences, 7th edition

\title{
Significance of Software Development Models in Current Context
}

\author{
Praveen Kumar, Ajit Singh \\ Department of MCA, Patna Women's College (Autonomous)
}

Patna University, Patna, India

Abstract: As we know with the advancement of a various software development models over the past years, it became a subject of an extreme interest to a reason and segregates them relying upon the applications, favorable circumstances and inconveniences. There are different elements that influence the software development activities; they must even be taken care of once we choose a development model. Several software products come to fail because of reasons like an associate unskilled developer, a time limit, a poor quality, a less client association and considerably more. A software development models ought to be selected with wisdom watching the conditions, and a quality of the developer, a user, a time and the complexes of the project. Of these factors play an important role within the success of the project. A software development models will be categorized as lightweight models and hefty weight models. This paper discusses various models on completely different metrics with execs associated cons of each of them and additionally facilitate to choose an acceptable model relying upon the project.

Keywords: Software Development Models, Spiral model, Waterfall model, Agile Model, RAD Model

\section{Introduction}

The methodology meant for a software development life cycle is really a structure used for coming up with and dominant the procedure of making a specialised software system to attain the specified goals. These innovative ways highlight the method of building software package at each stage of development. The fact is that, this progressive development is additional associated with project management and doesn't involve the utilization of any technicalities. There is additional concerning about proper planning and coming up with any 
iteration needed for building extremely scalable software system [1]. The simple determination of those principles is to build customised software package development as per the wants and one thing which each and every software package development team ought to adopt.

\section{Spiral Model:}

The Spiral model puts concentration on thorough risk assessment.Being a extremely refined style, it is meant to cut back the first risks within the project. so, you will got to have interaction folks with a robust background in risk analysis. As per the software package development method going by, the developers initiate on a smaller level and explore the enclosed risks in it. Further to the present, the developers are meant towards crafting a thought for iterating the spiral [2]. The accomplishment of any Spiral Lifecycle model is predicated on consistent, observant, and familiar management of the project.



Figure 1 : Spiral Model

Advantage:

1. Risk factors are significantly reduced

2. Large and complex software project can easily handled

3. We can add additional functionality later

4. Appropriate for extremely risky software project comes with varied business desires

Disadvantage:

1. Expensive model in software package development

2. Failure within the risk analysis part might harm the full Software project

3. Not acceptable for low-risk software project

4. Would possibly get never ending software project 


\section{Waterfall Model:}

If any one working in software package development company, at some purpose or the opposite, you'd might follow the water Model for a software product or project. Considered because the ancient software package development technique of explaining the software package development method in software package engineering, the water Development technique happens to clarify the method into a linear flow with a fixed sequence to let the users perceive that additional level is created progressive on completion of the previous one. Moreover, this technique additionally talks concerning the actual fact that going back to manage the changes is not doable.

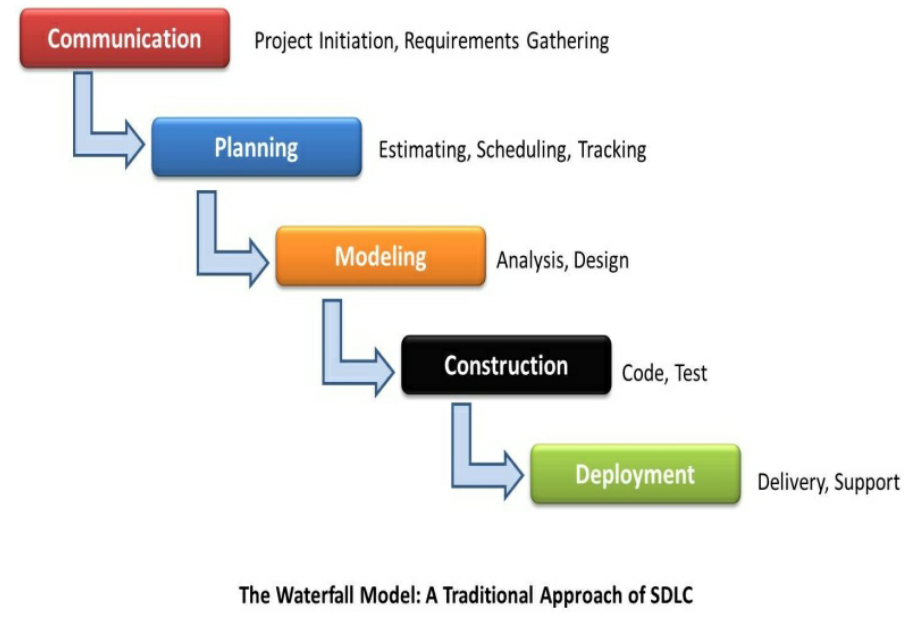

Figure 2 : Waterfall Model

Advantages:

1. Saves important quantity of your time simple to grasp and purposeful

2. easy enough to handle as model is rigid

3. Simple to grasp and purposeful

4. permits for simple testing and analysis

Disadvantages:

1. Not good for long and ongoing software project.

2. Not applicable for maintenance software project

3. No choice to grasp doable outcome of a software project

4. solely matches precise desires

\section{Agile Model:}

It is an innovative approach and one amongst the leading software package development models, the agile software package development methodology is employed for articulating a well-organized project management procedure providing repeated alterations.

Certainly, Agile development methodology is one theoretical define for enterprise many software projects [3]. Another smart factor concerning it's that it minimizes peril by making software package in brief time boxes, referred to as iterations, that happen to last to one 
month.

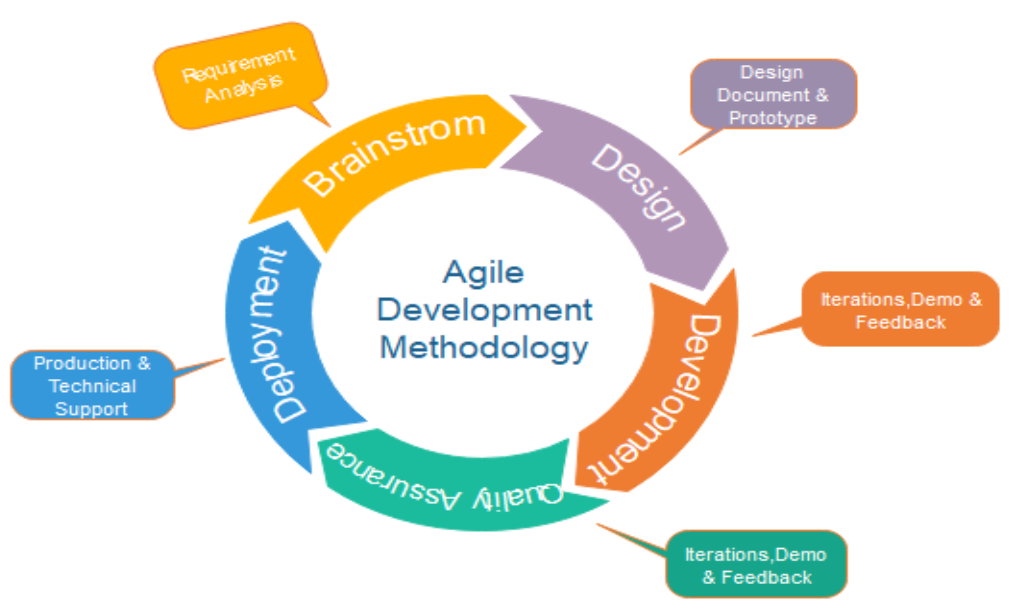

Fig. Agile Model

Figure 3 : Agile Model

Advantages:

1. Agile approaches in an reconciling method that responds to changes favourably

2. Permits for direct communication to keep up transparency

3. Continuous Improvement in quality by finding and fixing defects quickly and distinctive expectation mismatches early.

4. Resource requirement are minimum

5. Promotes teamwork

Disadvantages:

1. Focuses on operating with software package and lacks documentation potency

2. Possibilities of obtaining off-track because the outcome aren't clear

3. High individual dependency due to less documentation

4. Not good for large projects

\section{RAD Model:}

This model provide fast results, fast application development could be a software package development model that is supposed to relinquish glorious processes with the help of alternative software package approaches. It is created to require the most advantage from the event software package [4]. Undoubtedly, it's designed to enhance the workability of the full software or web application project for highlight the participation of an active user.

Advantages:

1. Makes the complete development method easy

2. Assists consumer in taking fast reviews

3. Encourages feedback from customers for improvement

4. Progress can be measure. 
Disadvantages:

1. contingent on the team for performance

2. Works on modularized system confined on this technique

3. Needs very sure-handed personnel to handle complexities

4. Not applicable for the little budgeted projects

5. Reusability of components inhanced

\section{Prototype Model:}

It is a specialised software package development principle that initiates developers towards creating solely the sample of the resolution to validate its purposeful essence to the customer and build essential iteration before making the authentic final product and also the final QA testing. The most effective part of this technique is that it tends to resolve a group of diversifying problems occurring with the waterfall method [6].

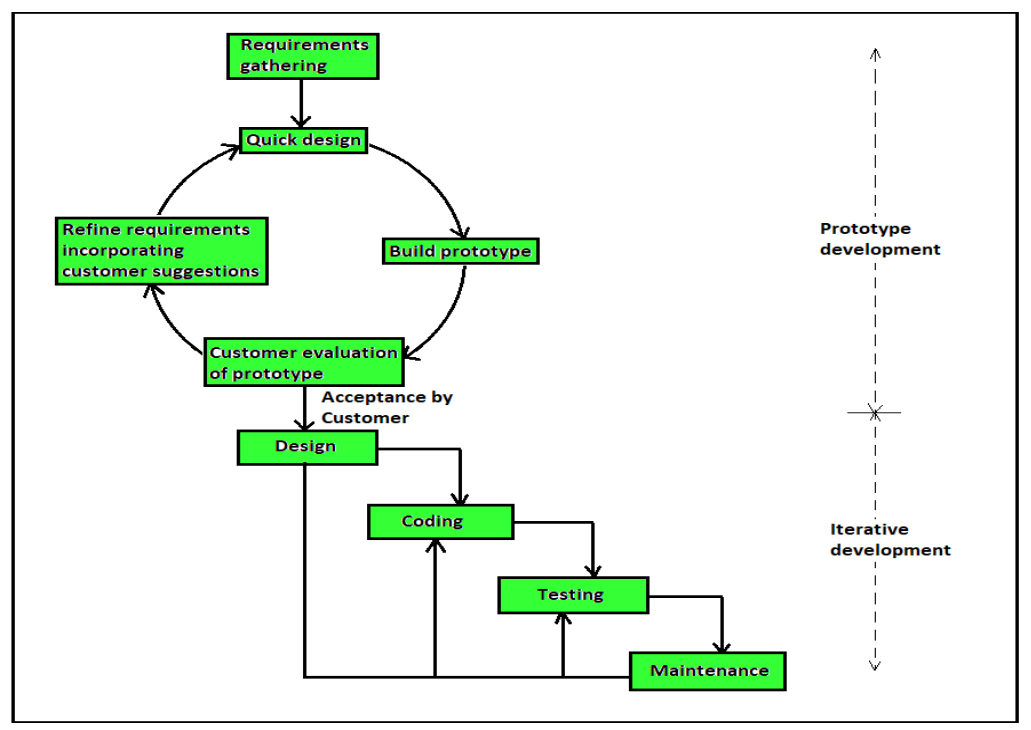

Figure 4: Prototype Model

Advantages:

1. provides a transparent plan concerning the purposeful method of the software package

2. Reduces the danger of failure in an exceedingly software package practicality

3. Assists well in demand gathering and also the overall analysis

4. this is cost effective model

Disadvantages:

1. possibilities of extension in managerial cost

2. Excessive involvement of consumer will have an effect on process

3. Too several changes have an effect on the advancement of the software package 


\section{Dynamic System Development Model:}

Authentically developed and derived from the fast application development methodology, it's an reiterative and progressive approach that focuses on the involvement of the user.

The task of this technique or principle is to supply software package development advancement among the required timeframe and also the allotted budget.

The terribly reason why it's quite in demand within the world of software package development.

\section{Advantages:}

1. Users obtaining a position of the software package development method

2. practicality deliverables are fast

3. Offers easy accessibility to end-users by the developers

Disadvantages:

1. this technique is dear to implement

2. Not appropriate for little organizations

\section{Extreme Programming Model:}

Extreme programming is known by the actual fact that client involvement within the software package building method is implausibly high. It is an agile software package engineering methodology, extreme programming methodology is presently referred to as XP methodology. It is primarily used for crafting software package among a really unbalanced atmosphere [7]. It allows bigger tractableness among the modeling procedure.

The foremost aim of this XP model is to cut back the price of software package essentialities. It is fairly mutual within the XP model that the worth of sterilization the wants on future stages within the project is very whooping.

\section{Advantages:}

1. It lays specialise in client involvement

2. Establishes rational plans and schedules

3. Developers ar exceptionally committed to the project

4. Equipped with stylish ways for quality software package

Disadvantages:

1. Effectiveness depends on the folks concerned

2. needs frequent meeting for development raising total prices

3. Necessitates for excessive development changes

4. actual potentialities and future outcomes ar very unknown 


\section{Feature Driven Model:}

It is an reiterative methodology for software package development, it's aimed toward serving an oversized variety of groups functioning on a project supported object-oriented technology this type of model is tight for corporations that ar passing on from a phase-based technique to AN reiterative approach. It is already referred to as AN FDD methodology and is extremely purposeful and artistic enough to manage varied complexities.

\section{Advantages:}

1. Moves larger comes with continuous success

2. the simplest five procedures bring outcome in an exceedingly higher manner

3. designed on pre-set standards of software package development, it's programmed for simple execution

4. comes that require continuous updates ar powered by a feature-driven methodology that ensures all desires ar taken care of.

5. ends up in options that continually outshine the inputs

6. Since this is often supported a number of the most effective software package building practices, any developer with relevant expertise will handle and manage project-related tasks with ease.

\section{Disadvantages:}

1. Not appropriate for smaller comes and one developer - continually a large team is needed, which implies that we tend to cannot ever guarantee a brisk point in time unleash.

2. extremely dependable on the leading developers, necessitating for the whole structure the method must be monitored through every part as even a moment flaw will produce chaos within the system.

3. extremely dependable on the leading developers, necessitating for the whole structure the method must be monitored through every part as even a moment flaw will produce chaos within the system.

\section{Rational Unified Process model:}

Smartly known as RUP, Rational Unified process model powers software package development exploitation rational tools. This methodology segregates the growth method into four completely different stages that every embrace business modeling, scrutiny and style, enactment, testing, and disposition. This is an object-based and web-empowered program growth methodology. The model tends to help software package developers in stating tips, templates, and specimens for all options and stages of software package development [8]. 


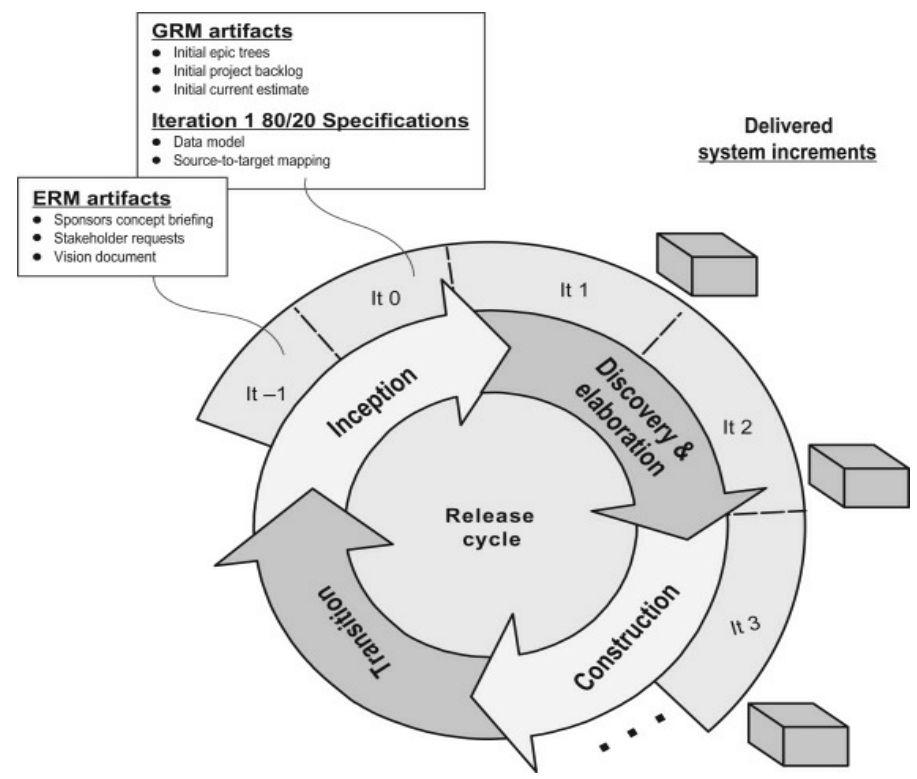

Figure 4: Rational Model

Advantages:

1. Lays high specialise in precise documentation

2. Removes project risks joined with consumer evolving desires

3. terribly less demand for integration

Disadvantages:

1. desires overly skilled software package developer

2. the event procedure of the methodology is sophisticated

3. Integration would possibly cause confusion

4. terribly sophisticated to grasp

\section{Scrum Development Model}

SCRUM is that the most generally most popular agile development approach for many software package teams- that is really AN agile framework. (Likewise, KANBAN could be a method that helps groups to collaborate and work effectively.) essentially, this glorious methodology is appropriate for those software package comes that are perpetually sterilization or having very scaling needs. The scrummage software package development model initiates with transient coming up with, conference and completes with a last review. This methodology is employed for building software package that happens to incorporate a series of iterations to get the desired software package. It is an ideal approach as a result of it effortlessly brings on course the deliberate progressing comes.

\section{Advantages:}

1. Deciding lies within the hands of the team

2. Business demand document is taken into account insignificant

3. Gently controlled technique empathizing with constant change 
Disadvantages:

1. The process technique suffers attributable to wavering prices

2. Not appropriate for giant sized comes

3. needs extremely skilled team, that has no place for novices

\section{Conclusion:}

Technology has paved method for several forceful changes in software package development that even have modified the approaches to software package programming.

The main factor during this side is that it deals with a spread of complexities, that need skilled handling. Software development has fixed methodologies that job on bound platforms, that permits them the liberty to work [10]. This concerns high-quality performance beneath the steering of pros, UN agency have years of expertise in handling technical problems efficiently. With varied sorts of methodologies applicable to a unique set of software package development comes, the developers have various choices to make excellently operating software package.

\section{REFERENCES:}

[1] www.ijcsi.org/papers/7-5-94-101.pdf

[2] Ian Sommerville, "Software Engineering", Addison Wesley, 7th edition, 2004.

[3] United States, Navy Mathematical Computing Advisory Panel (29 June 1956),Symposium on advanced programming methods for digital computers, [Washington,

D.C.]: Office of Naval Research, Dept. of the Navy, OCLC 10794738

[4] Benington, Herbert D. (1 October 1983). "Production of Large Computer Programs"(PDF). IEEE Annals of the History of Computing (IEEE Educational Activities

Department) 5(4): 350- 361. doi:10.1109/MAHC.1983.10102. Retrieved 2011-03-21.

[5] Royce, Winston (1970), "Managing the Development of Large Software Systems"(PDF), Proceedings of IEEE WESCON 26 (August): 1-9

[6] Wasserfallmodell > Entstehungskontext, Markus Rerych, Institut für Gestaltungs- und Wirkungsforschung, TU-Wien. Retrieved on 2007-11-28 fromhttp://cartoon.iguw.tuwien.ac.at/fit/fit01/wasserfall/entstehung.html

[7] Larman, Craig (June 2003). "Iterative and Incremental Development: A Brief History" (PDF). Computer 36 (6):47-56.doi:10.1109/MC.2003.1204375. ISSN 0018-9162 
[8] DOD-STD-2167 Defense Systems Software Development (04 JUN 1985) on everyspec.com

[9] Rlewallen, "Software Development Life Cycle Models", 2005 ,http://codebeter.com.

[10] Karlm, "Software Lifecycle Models', KTH,2006.

[11] Kevin Forsberg and Harold Mooz, "The Relationship of System Engineering to the Project Cycle," in Proceedings of the First Annual Symposium of National Council on System Engineering, October 1991: 57-6

[12] Boehm B, "A Spiral Model of Software Development and Enhancement", ACM SIGSOFT Software Engineering Notes, ACM, 11(4):14-24, August 1986 